\title{
L'appareil glandulaire de la tête du chameau
}

\author{
par M. A.F. TAYEB, M.V.SC. \\ College of veterinary medicine, Fouad I University, Giza (Egypt)
}

Traduction : P.-C. BLIN

Chef de Travaux d'Anatomje à l'École Nationale Vétérinaire d'Alfort

\section{INTRODUCTION}

IL n'existe que peu de renseignements sur l'anatomie de la tête du chameau. Pourtant. son étude et plus particulièrement celle de son appareil glandulaire apporterait une intéressante contribution dans le domaine de l'anatomie et de la pathologie comparatives.

CHAUVEAU (1891) présente la glande parotide du chameau comme ressemblant à celle du bœuf; toutefois, elle s'étend plus dans le sens horizontal que dans le sens vertical. Le canal de Sténon croise la surface du masséter pour gagner sa destination.

La glande sous-maxillaire est faiblement lobulée; le canal de Wharton ne s'ouvre pas au sommet d'un barbillon mais à la base du frein de la langue par une sorte d'orifice aréolé percé à fleur de muqueuse.

LESBRE (1903) décrit la parotide du chameau. comme celle du bøuf quant à sa forme et à sa disposition. Mais sa couleur est plus foncée chez le chameau, vu qu'elle est aussi rouge que le masséter avoisinant. Elle pèse de 180 à 190 grammes et ne descend pas au-dessous du niveau de l'aile de l'atlas. Le canal de Sténon est de tout petit calibre et croise la surface du masseter pour s'ouvrir en regard de la deuxième prémolaire supérieure.

La glande sous-maxillaire est un disque triangulaire situé au-dessous de l'aile de l'atlas.

Elle pèse de 65 à 70 grammes. Le canal de Wharton est fort. La glande sublinguale est mince et allongée. Ses canaux excréteurs viennent deboucher au fond du canal lingual dans les intervalles de la série des papilles que l'on trouve à cet endroit.

LEESE (1927) indique que la parotide est petite, que le canal de Sténon s'ouvre en regard de la troisième molaire supérieure. La glande sousmaxillaire est située sous la parothde et la veine jugulaire; elle est aplatie, de forme circulaire.

La glande sublinguale est insignifiante.

La glande occipitale n'existe que chez le mâle; elle est située dans la peau à 10 centimetres audessous de l'occiput, en région nuchale, et forme deux amas situés de chaque côté de la ligne médiane. Chaque amas couvre une surface de 23 à 24 centimètres carrés.
Le ganglion sous-maxillaire est situé en arrière de l'angle de la mâchoire; il s'explore aisément chez l'animal vivant. Les ganglions pharyngiens se trouvent au-dessous de la glande sous-maxillaire. Le ganglion principal est aplati et mesure $7 \mathrm{~cm} .5$ de long.

\section{MATÉRIEL D'ÉTUDE ET MÉTHODE}

Cette étude porte sur sept têtes prélevées aux abattoirs du Caire et injectees par l'artère carotide primitive avec une solution de formol à $10 \%$.

La parotide et la sous-maxillaire furent, chaque fois, pesées et mesurées à l'état frais.

\section{L'APPAREIL GLANDULAIRE DE LA TÊTE DU CHAMEAU}

L'appareil glandulaire de la tête du chameau comprend l'appareil salivaire, la glande occipitale, les ganglions lymphatiques; la thyroïde est décrite à propos de l'étude des voies respiratoires; les glandes muqueuses à propos des cavités buccale et pharyngée.

\section{Les glandes salivaires.}

La parotide. - Elle est de forme ovale, mais elle est légèrement crénelée à son bord antérieur. Elle est de coloration brun rougeâtre et pèse 130 à 140 grammes. Sa mensuration donne : Iongueur : 15 à 16 centimètres; largeur : 8 à 9 centimètres; epaisseur : $2 \mathrm{~cm} .5$.

Elle couvre une grande surface du masséter et se trouve située plus superficiellement que chez le cheval et le bouf, étant rejetée en dehors par suite de la présence de la glande sous-maxillaire entre elle et l'atlas.

Sa face externe est convexe, le plus souvent en position sous-cutané: ce n'est qu'en partie inférieure qu'elle est recouverte par le risorius.

Le muscle parotido-auriculaire est très petit.

Un gros ganglion Iymphatique, le ganglion parotidien, ayant 2 à $2 \mathrm{~cm} .5$ de longueur, est serti dans une profonde dépression du bord antérieur de la glande et est en rapport avec l'arcade zygomatique. 
Un autre ganglion lymphatique, plus petit, existe au bord postérieur de la parotide.

Le bord antérieur est mince, irrégulièrement convexe, et présente les crénelures déjà mentionnées.

Le bord postérieur est épais, plus régulièrement convexe.

La face profonde de la glande est en rapport avec le tendon intermédiaire du digastrique et le muscle occipito-mandibulaire, les artères temporale et faciale, le nerf facial et ses divisions.

Ia veine jugulaire passe sous la parotide sans la traverser.

En région postérieure, la face profonde de la parotide est en rapport avec la glande sous-maxillaire. Quelquefois, le tendon du muscle sterno-mastoïdien sépare en partie les deux glandes, s'il atteint l'apophyse mastoide.

L'extrémité supérieure de la glande est profondément échancrée pour recevoir la base de la conque.

L'extrémité inférieure ou apex est ordinairement pointue mais, sur quelques sujets, nous l'avons trouvée échancrée pour recevoir l'extrémité supérieure du ganglion sous-maxillaire.

L'apex est en rapport, à la surface du masséter, avec la veine faciale et la branche montante du maxillaire inférieur.

La parotide est très lobulée; les lobules sont très fins; ils sont masqués par la présence d'une nappe de tissu conjonctif épaisse et opaque qui les entoure.

Le canal de Sténon. - A son origine, ce canal a un fort calibre (I centimètre de diamètre environ); celui-ci décroît progressivement jusqu'à sa terminaison. Il se forme immédiatement sous la face interne de la glande parotide par la réunion de trois ou quatre canalicules. L'un d'eux émerge sous le bord antérieur de la glande, au niveau d'un point situé entre le tiers moyen et le tiers inférieur de ce bord, se dirige vers l'avant, d'abord de haut en bas puis de bas en haut en formant une anse cachée par le risorius, mais couvrant le masséter.

Au voisinage du bord antérieur du masséter, le canal de Sténon devient superficiel et facilement accessible; il se dispose alors dans l'espace situé entre l'artère faciale inférieurement et le nerf buccal supérieur supérieurement.

Au bord antérieur du masséter, il croise la veine faciale en dedans, traverse les glandes buccales près du bord supérieur du buccinateur pour s'ouvrir au milieu d'une papille aplatie, ayant l'aspect d'une corolle, en regard de la troisième molaire supérieure.

La glande parotide est irriguée par les artères faciale, auriculo-méningée et auriculaire postérieure.

Les veines se déversent toutes dans'la grande veine auriculaire qui traverse la glande de haut en bas et de l'extérieur vers l'intérieur pour se jeter, au niveau du tiers inférieur de la face profonde de la parotide, dans la veine jugulaire.

La glande sous-maxillaire. - Cette glande rie mérite pas son nom : elle est située entièrement en dehors de la mâchoire, dans l'espace compris entre l'aile de l'atlas en haut, l'occipito-mandibulaire en avant et en bas et la partie postérieure de la glande parotide en dehors, reposant sur l'aile de l'atlas par son extrémité supérieure, la séparant de la glande parotide.

Par sa forme, la glande sous-maxillaire rappelle une oreille humaine mais, chez. quelques sujets, elle est discoïde, avec une extrémité inférieure allongée et pointue ou arrondie.

Elle est de couleur jaune pâle ou jaune sale, pèse 60 à 70 grammes environ et fait 11 à. 12 centimètros de longueur, 7 à 9 centimètres de largeur et 2 centimètres à $2 \mathrm{~cm}$. 5 d'épaisseur. Ses lobules sont plus gros yue ceux de la parotide. Sa face exlerne est légèrement convexe, le plus souvent en relation avec la parotide.

La face profonde est plane, en relation avec les ganglions rétro-pharyngiens,-..-La veine occipitale passe entre les deux glandes.

Le bord antérieur est épais et directement en rapport avec l'occipito-mandibulaire; le bord postérieur est plus mince, convexe dans sa partie supérieure, rectiligne inférieurement.

L'extrémité supérieure est mince et arrondie; elle est sous-cutanée en dehors et repose sur l'aile de l'atlas en dedans.

L'extrémité inférieure est étroite, arrondie ou pointue et sise en arrière de l'angle du maxillaire inférieur, en rapport avec la veine jugulaire, là où elle reçoit les trois branches principales qui drainent le sang de la tète.

Le canal de Wharton. - Ce canal est de calibre moyen : 4-5 millimètres de diamètre environ. Il commence au milieu du bord antérieur de la glande sous-maxillaire, au-dessous du point de pénétration de l'artère dorsale de cette glande, se dirige en avant entre l'occipito-mandibulaire et le muscle ptérygoidien interne, s'incurvant de haut en bas et d'arrière en avant à la face antérieure du digastrique et croisant le muscle stylo-hyoïdien et le stylo-hyal. Plus en avant, il longe la face externe du styloglosse, prenant une direction extérieure et ventrale jusqu'à la glande sublinguale.

Il se termine à $2-2 \mathrm{~cm}, 5$ du frein de la langue par ur orifice de 1 millimètre de diamètre environ.

Avant de se terminer, le canal de Wharton s'élargit brusquement et forme une sorte d'ampoule.

La glande sous-maxillaire est irriguée par l'artère carotide primitive et par l'artère occipitale; quelquefois la branche dorsale vient de la carotide externe 
juste en avant de la bifurcation carotidienne.

La glande sublinguale. - Elle ressemble à celle du cheval, mais elle est moins développée; ses lobules sont plus fins, lâchement réunis les uns aux autres; les canaux excrèteurs sont en moindre nombre.
Chạue amas comprend des petits lobules étroitement associés, très adhérents à la face interne du tegument, s'étendant à partir d'un point situé près de la iigne médiane, à 10 centimètres en arrière de la protubérance occipitale, jusqu'à ún point situé à 2 centimetres en arrière de la conque auriculaire.

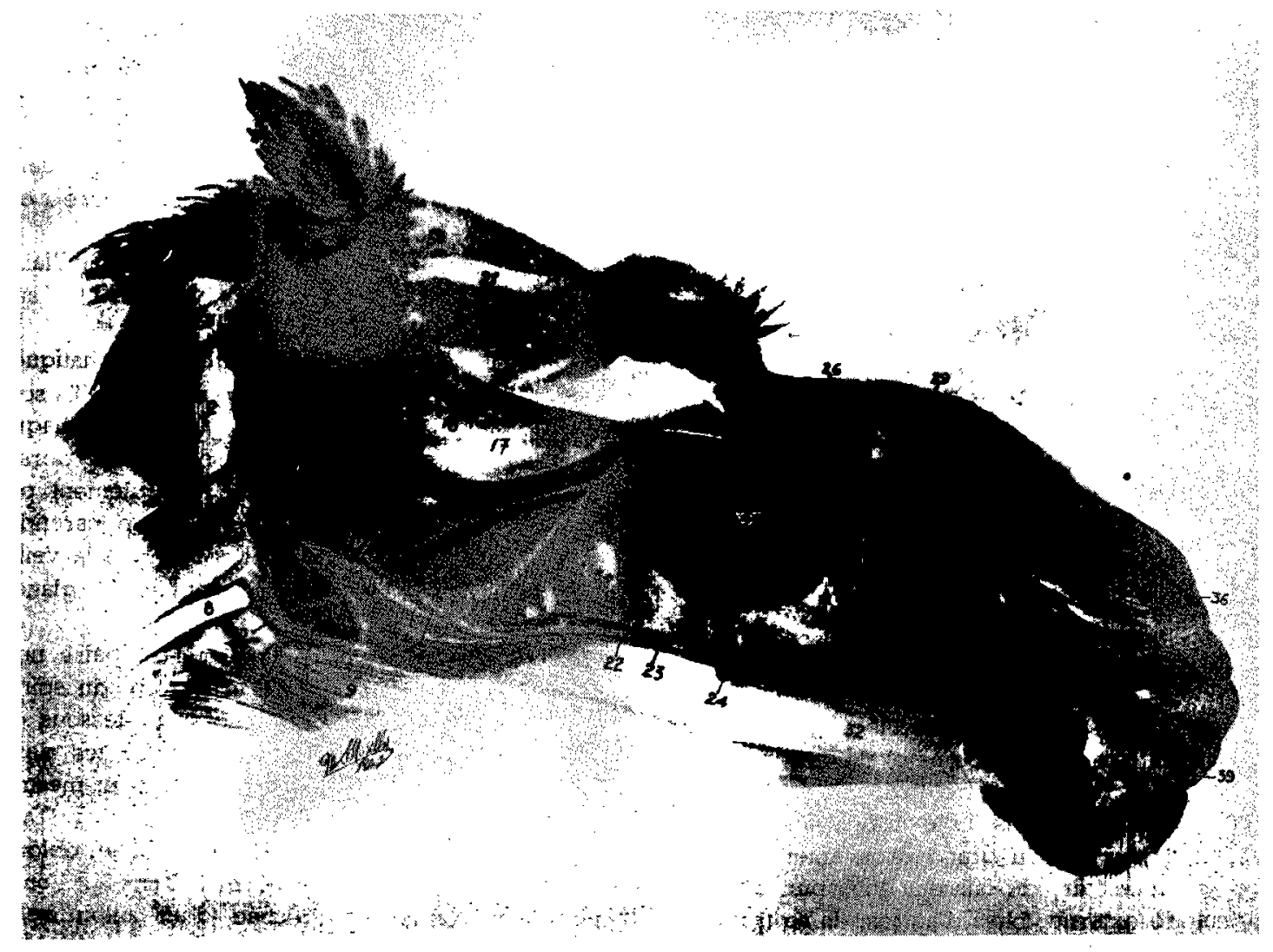

Figure III. - Tête du Chameau (cồté droit).

1. Parotide. - E. Apex. - B. Base. - I. Ganglion parotidien.2. Glande sous-maxillaire. - 3. Ganglion lymphatique sur Je tendon du grand droit antérieur de la téte. - 4. Grand droit antérieur de Ja tête. - 5. Complezus. - 6. Ganglion sousmaxillaire. 1. Veine jugulaire. - 8. Sterno-céphalique. -

La glande zygomatique. - Par sa position el par sa situation, elle rappelle celle du chien. C'est soit une glande unique, de forme arrondie, ayant un seul canal excréteur, soit un groupe glandulaire comparable à celui du chien. Les canaux s'ouvrent alors dans la partie postérieure et supérieure de la cavité buccale.

\section{Iu glande occipitale du chamean.}

On ne la rencontre que chez le mâle. Elle est faite de deux amas situés chacun latéralement par rapport à la ligne occipitale médiane.
9. Omo-hysidien. - 10. Veine linguale. - 11. Veine faciale. 30. Canin. - 34. La glande malaire inférieure, très développée, visible a travers le tendon semi-transparent du muscle buccinateur. - 35. Orbiculaire des paupieres. - N. Insertion labiale du muscle releveur commun de l'aile du nez et de la lèvre supérieure. - 40. Buccinateur. - M. Portion du malaire. -

R. Fragment du risorius. - S. Fragment du zygomatique.

Le grand axe de la glande affecte une orientation antéro-externe et mesure 10 centimètres eriviron. La largeur est de 2 à $2 \mathrm{~cm}$. 5 .

La forte capsule fibreuse qui entoure la glande envoie de nombreuses cloisons à son intérieur. cloisons qui enserrent étroitement chaque lobule.

La glande offre plusieurs fins orifices à la surface du tégument. Elle est irriguée à partir de l'artère occipitale.

\section{L'appareil lymphatique.}

Un trait caractéristique est la présence de 


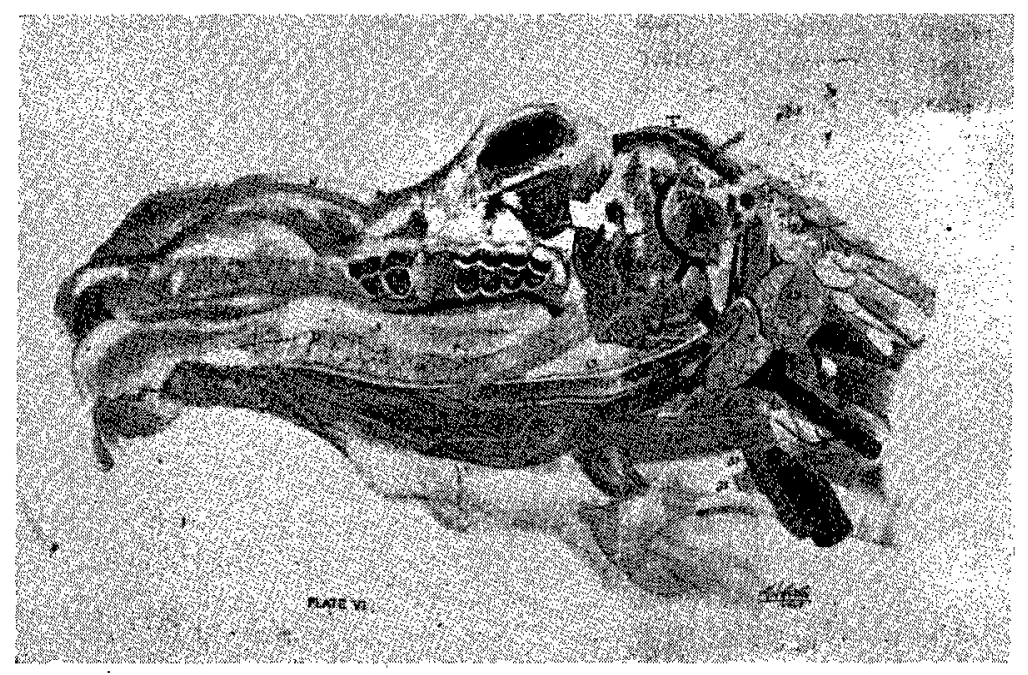

Figure IV. - Tête du Chameau. Région ptérygoídienne.

13. Canal de Wharton. - 17. Nerf grand hypoglosse. - 25. Glande sous-maxillaire. 26. Tendon intermédiaire du digastrique et de l'occipito-mandibulaire. - 27. Muscle occipitomandibulaire. - 46. Glande sublinguale, - D. Canaux excréteurs. - P. Papilles sur lesquelles s'ouvrent les canaux. - L. Ganglions rétropharyngiens. - O. Éléments osseux incrustés dans les tendons des muscles.

nombreux petits ganglions lymphatiques situés ordinairement au voisinage de l'insertion des muscles de la nuque et de la région maxillaire externe.

Ces ganglions sont de forme ovale ou ronde et ont un diamètre de 1 centimètre environ.

Chez quelques sujets, ils existaient au niveau du tendon d'insertion du grand droit antérieur de la tête, du masséter, du faisccau inférieur du scutoauriculaire externe; chez d'autres, ils se trouvaient au niveau du zygomatique et du scutellaire; on ne les rencontrait pas aux endroits signalés plus haul. Dans un autre cas, ils existaient sur chaque muscle, sauf au niveau du scutellaire; il y en avait trois de chaque côté de la crête pariêtale.

Ainsi, la position des ganglions 'lymphatiques de la tête est très variable et diffère suivant les sujets; seuls, les ganglions rétro-pharyngiens, sous-maxillaire et préparotidien ont une position constante. Dans quelques cas, des parcelles osseuses de dimensions et de forme variées rappelant apparemment des ganglions furent trouvées incrustées danis les tendons des muscles de la nuque, mais à la section, on constata qu'il s'agissait de tissu osseux.

Le ganglion sous-maxillaire. - Il est situé en arrière de l'angle de la mâchoire, enclavé entre l'occipito-mandibulaire en dehors et l'omo-hyoïdien en dedans. Le ganglion est volumineux; il a la forme d'un croissant avec une extrémité supéricure assez pointue; il est de couleur brun grisâtre et mesure environ 0 à 7 centimètres de long, 2 à 3 centimètres de large et $2 \mathrm{~cm} .2$ à $2 \mathrm{~cm} .5$ d'épaisseur. Sa face convexe est orientée vers l'arrière et son bord est en rapport avec la veine faciale en dehors et la veine linguale en dedans.

L'extrémité supérieure est engagée dans la jonction de la veine jugulaire avec les veines faciale et linguale. L'extrémité inférieure est libre

Le ganglion sous-maxillaire s'explore facilement sur l'animal vivant.

Les ganglions lymphatiques rétro-pharyngiens. - Ils sont au nombre de deux de chaque côté. Ils sont situés dorsalement et ventralement par rapport à l'artère carotide, séparant celle-ci de la veine occipitale : et de la glande sous-maxillaire.

La branche ventrale de la première paire nerveuse cervicale et le rameau accessoire du spinal croisent l'artère carotide en dehors, au-dessous du ganglion ventral ou, quelquefois, entre les deux ganglions. Chaque ganglion est ovalaire et mesure $3 \mathrm{~cm} . \times 2 \mathrm{~cm} . \times 0 \mathrm{~cm} .5$.

Le ganglion ventral est de forme variable, oblong sur quelquas sujets ct mesure $4 \mathrm{~cm} . \times 2 \mathrm{~cm} .5 \times 1 \mathrm{~cm}$. : sa face profonde est creusée d'un sillon relativement profond pour le passage de l'artère carolide.

\section{RÉSUMÉ}

L'appareil glandulaire de la tête du chameau a été étudié sur sept pièces prélevées aux abattoirs du Caire. Par ailleurs, plusieurs mensurations ont été faites à l'état frais.

1. La parotide est étendue, irrégulièrement allongée, de forme ovale, de couleur brun rougeâtre. Elle est située plus superficiellement que chez, les autres animaux domestiques.

2. Le canal de Sténon est de fort calibre, passe à la surface du masséter et s'ouvre en regard de la troisième molaire supérieure sur une papille aplatie en forme de corolle.

3. La glande sous-maxillaire est jaune pâle et à la forme d'une oreille humaine ou d'un disque triangulaire. Elle n'est pas sous-maxillaire par sa position 
et repose par son extrémité supérieure sur l'aile de l'atlas.

4. Le canal de Wharton est long et de fort calibre; son ouverture se fait en arrière du frein de la langue.

5. La glande occipitale n'existe que chez le mâle. Elle est formée de deux amas de petits lobules qui adhèrent intimement au tégument de la région occipitale.

6. Je ganglion sous-maxillaire est situé en arrière de l'angle de la mâchoire; il est étendu, facilement explorable sur :'animal vivant.
7. Les ganglions lymphatiques de la région pharyngée sont de position supra-pharyngienne; le ganglion ventral est ordinairement important et de forme oblongue.

8. De nombreux ganglions lymphatiques sont remarqués a l'entour des muscles de la région occipitale mais leur position est très variable.

9. Chez quelques sujets, de nombreux corpuscules d'origine osseuse, simulant des ganglions, ont été trouvés au niveau des muscles de là régrion occipitale. 